\title{
Unidimensionality Versus Statistical Accuracy: A Note on Bejar's Method for Detecting Dimensionality of Achievement Tests
}

\author{
Michelle Liou

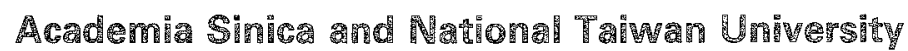

\begin{abstract}
A simulation investigated use of the difficulty parameter (Bejar, 1980) to evaluate item unidimensionality. Artificial tests were designed to be nonequivalent in both length and dimensionality. Simulated item responses to the tests were analyzed with the LOGIST computer program. Two indices were calculated: the slope of the principal axis between the content-areabased item difficulty estimates and corresponding total-test-based estimates, and the correlation between the two sets of estimates. Results show that the magnitude of the correlation coefficient provides no information about dimensionality of a set of test items. The slope of the principal axis, on the other hand, is sensitive to multidimensionality in the data as well as test length. The size of the slope adequately detects the dimensionality of items for relatively long tests. Index terms: equating, item difficulty parameters, item response theory, unidimensionality.
\end{abstract}

Bejar (1980) proposed using content-area-based and total-rest-based item difficulty $(b)$ estimates to detect the dimensionality of achievement tests. Bejar argued that if a set of test items is unidimensional, then grouping test items into subareas to calibrate items will be irrelevant. Parameter estimates for items calibrated within different subareas, aside from sampling errors, should be identical; they should be close to a theoretical axis with a slope of 1 and an intercept of 0 . The Bejar procedure fits the principal axis to the content-area-

APPLIED PSYCHOLOGICAL MEASUREMENT

Vol. 12, No. 4, December 1988, pp. 381-386

(c) Copyright 1988 Applied Psychological Measurement Inc. 0146-6216/88/040381-06\$1.55 based $b$ values versus corresponding total-test-based values. The distance between the principal axis and the theoretical axis indicates how well the data satisfy the unidimensionality assumption of the logistic models.

Because $b$ parameters are generally estimated with less sampling error than are ability or item discrimination parameters, Bejar's method uses $b$ estimates to analyze dimensionality. However, the slope and intercept of the theoretical axis selected in the method show that the interpretation is unique for $b$ values calibrated with the total test and with the content areas.

The $\mathbb{R} T$ parameter scale has an indeterminacy problem that is particularly associated with the threeparameter logistic model. The same $b$ parameters calibrated with two separate datasets will scatter around a straight line (Lord, 1980, p. 36) that does not necessarily have a slope of 1 and an intercept of 0 . Nevertheless, test equating techniques are often used to generate comparability between the parameters.

When the same examinees' data are involved in both computer runs, as with Bejar's method, factors such as statistical accuracy as a result of test length and the deviation of item responses from the logistic function could also affect the calibration of item and $\theta$ parameters. Because these factors function differently in the calibration, the totaltest-based and content-area-based data are not likely to have the same meaning even if they are unidi- 
mensional. Consequently, these factors could also affect the sensitivity of Bejar's method in investigating the dimensionality of achievement tests.

In their subsequent study on the dimensionality of achievement tests, Hambleton and Rovinelli (1986) found that Bejar's method was insensitive to multidimensionality in the data except when the correlation between the underlying abilities was low and when a disproportionate number of test items measured one of the abilities. However, their findings were derived from a scale-free measure of association (the correlation) between the contentarea-based and total-test-based $b$ values.

In the present analyses, tests of unequal lengths were simulated to measure single and multiple latent traits. The simulated test data were calibrated with LOGIST 5 (Wingersky, Barton, \& Lord, 1983). The association between content-area-based and total-test-based $b$ values was evaluated by calculating both their principal axis and correlation. The present simulation results further highlight the sensitivity of Bejar's method to multidimensionality in the data.

\section{Method}

The simulation assumed that 1,000 examinees took all the simulated tests. $\theta_{1}$ for each examinee was generated from a standard normal distribution with the IMSL generator GGNPM (International Mathematical and Statistical Libraries, 1982) and was restricted to the interval from -2.5 to +2.5 . In the first evaluation, a pair of tests with 60 and 45 items were simulated for measuring a general factor. Each test consisted of three content areas. The 45-item test was comprised of items 1-15, 2135 , and 41-55 from the 60 -item test. The factor loading $p_{i \theta_{1}}$ of each item on the general factor was generated in an interval ranging from .6 to .7 by the IMSL generator GGUBS. A standardized latent response $y_{i}$ was simulated with a process described by Drasgow and Parsons (1983):

$y_{i}=\left(\theta_{1}\right) \rho_{i \theta_{1}}+e_{i}\left(1-\rho_{i \theta_{1}}^{2}\right)^{1 / 2}$,

where $e_{i}$ was a random deviate generated from a standard normal distribution. The item threshold values $\gamma$ for dichotomizing latent responses into binary scores were generated in an interval ranging from -1 to +1 . Guessing was simulated by generating item scores and then rescoring the 0 scores as correct with a probability of .15 , which was the hypothetical guessing parameter in the evaluation.

In the second evaluation, another pair of tests were simulated for measuring a general factor and three group factors. It was assumed that the group factors were uncorrelated with each other and with the general factor. Each simulation item also loaded only on a single group factor. The examinees' $\theta_{2}$, $\theta_{3}$, and $\theta_{4}$ on the group factors were independently generated from a standard normal distribution. Factor loadings $\rho_{i \theta_{2}}, \rho_{i \theta_{3}}$, and $\rho_{i \theta_{4}}$ of each item on the corresponding group factor were generated in an interval ranging from .3 to .5 . The communalities of the items were simulated to have comparable sizes in the two evaluations. Therefore, a multidimensional counterpart in Evaluation 2 al ways existed for each item in Evaluation 1. Latent responses to the multidimensional tests were also simulated with the method described by Drasgow and Parsons (1983):

$y_{i}=\sum_{j=1}^{4}\left(\theta_{j}\right) \rho_{i \theta_{j}}+e_{i}\left(1-\sum_{j} \rho_{i \theta_{j}}^{2}\right)^{1 / 2}$.

The item threshold values and the guessing parameter selected in Evaluation 1 were also used in Evaluation 2.

Item responses for the total test and for the subareas were calibrated in different runs with LOGIST 5 using default convergence criteria. The principal axis for the total-test-based and content-area-based $b$ values was also calculated. The sum of the squared distances was minimized in order to find the principal axis by

$d^{2}=\sum_{i}\left[\sin \phi\left(b_{i 1}-\bar{b}_{1}\right)-\cos \phi\left(b_{i 2}-\bar{b}_{2}\right)\right]^{2}$,

where $b_{i 1}$ was the $i$ th item content-area-based difficulty estimate and $b_{i 2}$ was the total-test-based estimate. The values $\bar{b}_{1}$ and $\bar{b}_{2}$ were corresponding mean difficulty estimates. The rotation of the axis locating the content-area-based estimate was through the angle $\phi$.

The slope of the principal axis was calculated from 


$$
\tan \phi=\frac{\left(\sigma_{1}^{2}-\sigma_{2}^{2}\right) \pm\left[\left(\sigma_{1}^{2}-\sigma_{2}^{2}\right)^{2}+4 \sigma_{12}^{2}\right]^{1 / 2}}{2 \sigma_{12}}
$$

(Bejar, 1980), where $\sigma_{1}^{2}$ and $\sigma_{2}^{2}$ were the variances of $b$ estimates and $\sigma_{12}$ was the covariance between the two sets of estimates. The value of $\tan \phi$ was selected as positive. The mean squared distance (MSD) was calculated by averaging the distances in Equation 3.

\section{Resulins}

The factor loadings and item threshold values used to obtain the response data are presented in Tables 1 and 2. The number of items $(N)$, mean, standard deviation and KR-20 for each simulation test and for its content areas are listed in Table 3. The value of tan $\phi$ for the principal axis and the correlation $r$ between difficulty parameters are also in Table 3. The slope of the principal axis for the total test was calculated by combining the contentarea-based $b$ values. Table 3 also shows the MSD for each content area from its corresponding principal axis. The plots of the content-area-based $b$ values and corresponding total-test-based values for the simulation tests are shown in Figures 1 and 2 .

The 60 - and 45 -item tests were simulated to be identical in all respects except for their lengths. The simulation results from Evaluation 1 indicate that the values of tan $\phi$ for the 45 -item tests differ from 1 even though the test is unidimensional (e.g., the third content area has a slope of 1.20 ). The $45-$ item test also has larger MSD values than the 60 item test.

In Evaluation 2, the pairs of tests were simulated as multidimensional. The slopes for the 60 - and 45 -item tests both deviate from 1 . However, the slope of the 60 -item test $(.878)$ is as discrepant as the slope for the 45-item test (1.088) in Evaluation 1. This result implies that the test length and multidimensionality in the data might both account for the deviation. For both evaluations, the correlations between the $b$ values fail to give any interesting pattern (all the correlations are greater than .9).

Table 1

Item Threshold Values $(\gamma)$ and Factor Loadings $\left(\rho_{\ell} \theta_{1}\right)$ for The Simulation Tests in Evaluation 1

\begin{tabular}{|c|c|c|c|c|c|c|c|c|}
\hline \multicolumn{3}{|c|}{ Content Area 1} & \multicolumn{3}{|c|}{ Content Area 2} & \multicolumn{3}{|c|}{ Content Area 3} \\
\hline Item & $\gamma$ & $\rho_{101}$ & Item & $\gamma$ & $\rho_{2} \theta_{1}$ & I tem & $\gamma$ & $P_{201}$ \\
\hline 1 & -.200 & .601 & 21 & .614 & .624 & 41 & .682 & .660 \\
\hline 2 & .154 & .620 & 22 & .342 & .611 & 42 & .071 & .647 \\
\hline 3 & .653 & .620 & 23 & -.173 & .678 & 43 & .150 & .687 \\
\hline 4 & -.469 & .653 & 24 & -.902 & .628 & 44 & .366 & .673 \\
\hline 5 & .766 & .621 & 25 & .179 & .635 & 45 & .218 & .698 \\
\hline 6 & -.502 & .623 & 26 & .441 & .641 & 46 & -.844 & .627 \\
\hline 7 & -.555 & .687 & 27 & .863 & .640 & 47 & -.247 & .658 \\
\hline 8 & -.736 & .604 & 28 & -.521 & .619 & 48 & -.475 & .605 \\
\hline 9 & .017 & .686 & 29 & .663 & .661 & 49 & -.694 & .665 \\
\hline 10 & -.602 & .695 & 30 & -.477 & .634 & 50 & -.340 & .661 \\
\hline 11 & -.962 & .620 & 31 & .381 & .637 & 51 & .232 & .664 \\
\hline 12 & .030 & .690 & 32 & -.633 & .662 & 52 & .909 & .700 \\
\hline 13 & .892 & .694 & 33 & -.049 & .657 & 53 & .549 & .631 \\
\hline 14 & .979 & .604 & 34 & -.989 & .666 & 54 & -.781 & .661 \\
\hline 15 & .966 & .619 & 35 &.- .282 & .632 & 55 & .373 & .627 \\
\hline 16 & -.296 & .687 & 36 & .545 & .634 & 56 & .662 & .623 \\
\hline 17 & .810 & .643 & 37 & .333 & .628 & 57 & -.839 & .636 \\
\hline 18 & -.781 & .621 & 38 & -.924 & .689 & 58 & .308 & .624 \\
\hline 19 & -.824 & .602 & 39 & .413 & .620 & 59 & .821 & .672 \\
\hline 20 & .959 & .614 & 40 & -.266 & .646 & 60 & -.774 & .653 \\
\hline
\end{tabular}




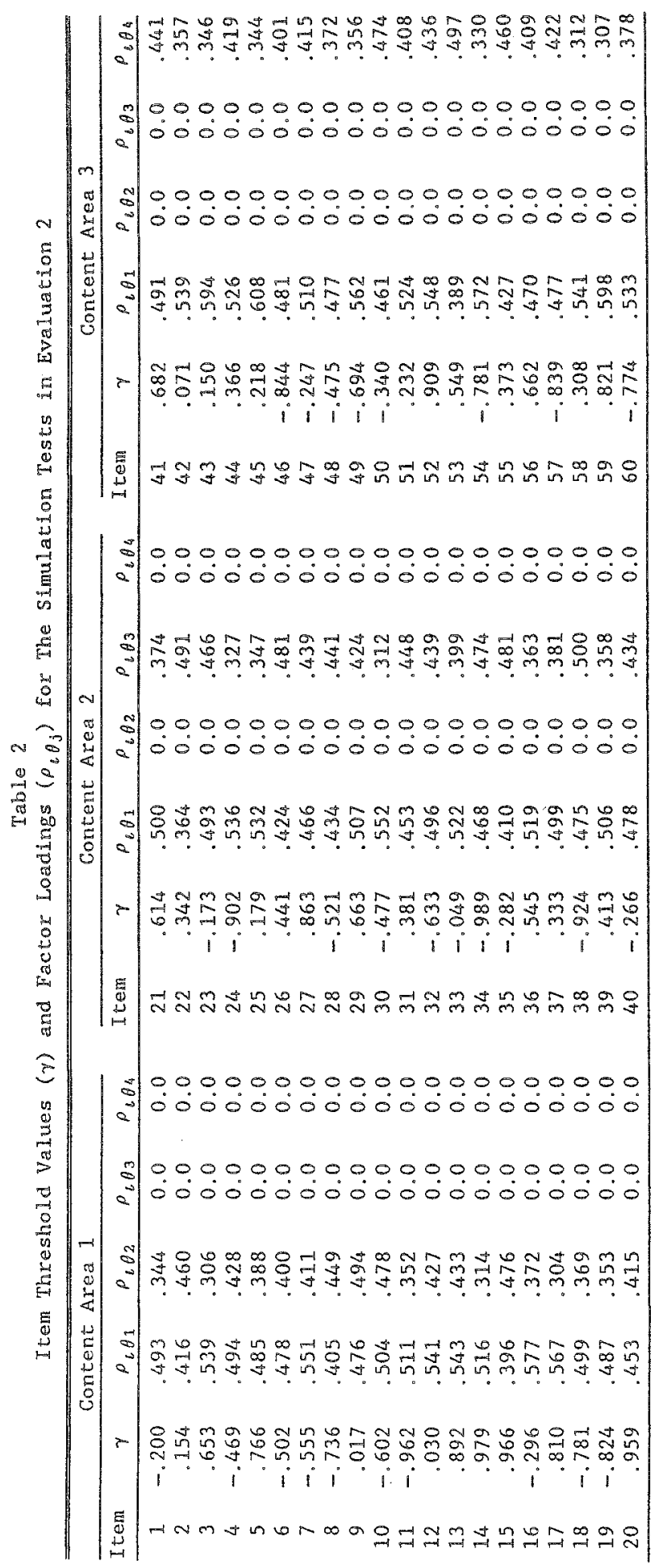

Downloaded from the Digital Conservancy at the University of Minnesota, http://purl.umn.edu/93227. May be reproduced with no cost by students and faculty for academic use. Non-academic reproduction requires payment of royalties through the Copyright Clearance Center, http://www.copyright.com/ 
Table 3

Content Area and Total Test Statistics for the 60-Item and 45-Item Tests in Evaluation 1 and Evaluation 2 for the Simulation Tests

\begin{tabular}{|c|c|c|c|c|c|c|c|c|}
\hline \multirow{3}{*}{$\begin{array}{l}\text { Test and } \\
\text { Statiotic }\end{array}$} & \multicolumn{4}{|c|}{ Evaluation 1} & \multicolumn{4}{|c|}{ Evaluation 2} \\
\hline & \multicolumn{3}{|c|}{ Content Area } & \multirow{2}{*}{$\begin{array}{l}\text { Total } \\
\text { Test }\end{array}$} & \multicolumn{3}{|c|}{ Content Area } & \multirow{2}{*}{$\begin{array}{l}\text { Total } \\
\text { Test }\end{array}$} \\
\hline & $\mathbb{1}$ & 2 & 3 & & 1 & 2 & 3 & \\
\hline \multicolumn{9}{|l|}{ Test 1} \\
\hline No. of Items & 20 & 20 & 20 & 60 & 20 & 20 & 20 & 60 \\
\hline Mean & 11.145 & 11.277 & 11.116 & 33.538 & 11.178 & 11.247 & 11.419 & 33.844 \\
\hline $\mathrm{SD}$ & 3.882 & 4.027 & 4.262 & 11.346 & 3.752 & 3.953 & 4.034 & 9.399 \\
\hline KR2O & .745 & .741 & .764 & .904 & .728 & .731 & .737 & .861 \\
\hline$r$ & .999 & .997 & .999 & .998 & .994 & .990 & .960 & .981 \\
\hline $\tan \theta$ & .962 & 1.045 & .993 & .995 & .915 & .824 & .882 & .878 \\
\hline MSD (Principal) & .004 & .005 & .001 & & .027 & .081 & .071 & \\
\hline \multicolumn{9}{|l|}{ Test 2} \\
\hline No, of Items & 15 & 15 & 15 & 15 & 15 & 15 & 15 & 45 \\
\hline Mean & 8.230 & 7.854 & 8.834 & 24.918 & 7.915 & 7.861 & 8.965 & 24.741 \\
\hline SD & 3.072 & 2.997 & 3.399 & 8.608 & 2.774 & 3.021 & 3.093 & 7.024 \\
\hline KR20 & .693 & .652 & .720 & .875 & .656 & .646 & .669 & .817 \\
\hline$r$ & .998 & .994 & .993 & .993 & .991 & .990 & .981 & .986 \\
\hline $\tan \theta$ & 1.075 & 1.030 & 1.200 & 1.088 & .995 & .904 & .859 & .925 \\
\hline MSD (Principal) & .012 & .008 & .048 & & .011 & .031 & .053 & \\
\hline
\end{tabular}

Higher

Plot of the $b$ Estimates for the 60-Item and 45-Item Tests in Evaluation 1

(a) 60 ltems

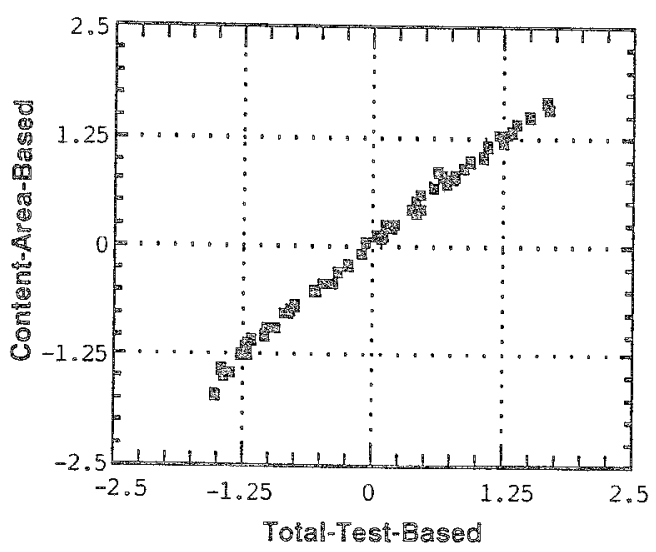

(b) 45 Items

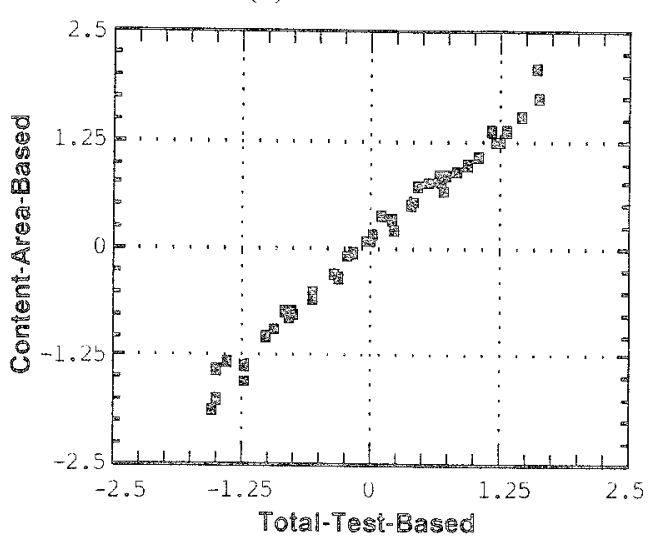

Downloaded from the Digital Conservancy at the University of Minnesota, http://purl.umn.edu/93227.

May be reproduced with no cost by students and faculty for academic use. Non-academic reproduction requires payment of royalties through the Copyright Clearance Center, http://www.copyright.com/ 
Figure 2

Plot of the $b$ Estimates for the 60-Item and 45-Item Tests in Evaluation 2

(a) 60 Items

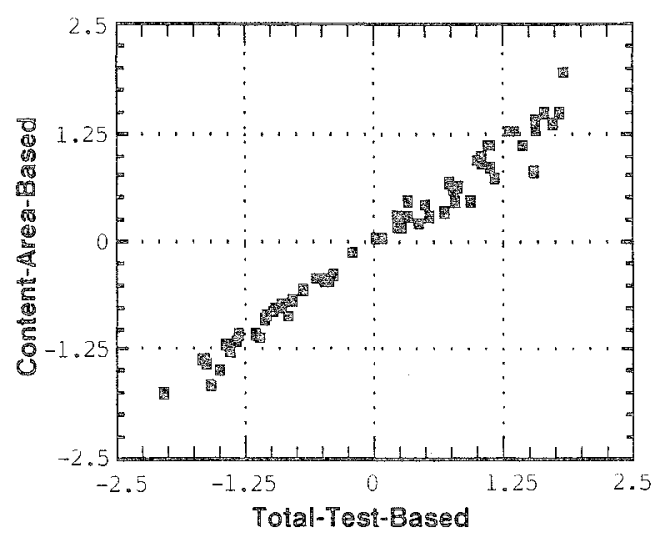

\section{Conclusions}

When applying Bejar's method for detecting dimensionality of achievement tests, researchers should be cautious when interpreting the slope of the principal axis. These results indicate that the size of the slope is sensitive to both multidimensionality and test length. With studies using empirical data, factors other than test length might also affect the magnitude of the slope, so that even more stipulations are needed before accepting the theoretical axis proposed by Bejar. On the other hand, Bejar's method worked reasonably well for the relatively long tests, which is illustrated by the fact that the size of the slope for the 60-item test in Evaluation 2 differed from 1 more than the same length test in Evaluation 1.

Bejar's method avoids the computational problem generally encountered with factor analyses of binary data. If the researcher accepts the threeparameter logistic model with some confidence and the content area test has a reasonable length (at least 20 items), the Bejar procedure provides a straightforward check on the unidimensionality of test data. The present results suggest the necessity of using other information from the data (e.g., test length, item-total correlations) in conjunction with (b) 45 Items

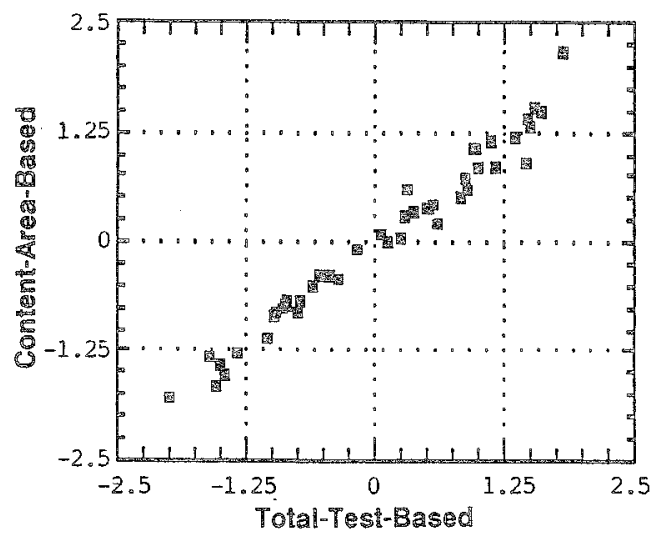

Bejar's method to address the question of item unidimensionality.

\section{RePerences}

Bejar, I. I. (1980). A procedure for investigating the unidimensionality of achievement tests based on item parameter estimates. Journal of Educational Measurement, 17, 283-296.

Drasgow, F., \& Parsons, C. K. (1983). Application of unidimensional item response theory models to multidimensional data. Applied Psychological Measurement, 7, 189-199.

Hambleton, R. K., \& Rovinelli, R. J. (1986). Assessing the dimensionality of a set of test tems. Applied Psychological Measurement, 10, 287-302.

International Mathematical and Statistical Libraries. (1982). IMSL Reference Manual (9th ed.). Houston TX: Author.

Lord, F. M. (1980). Application of item response theory to practical testing problems. Hillsdale NJ: Erlbaum.

Wingersky, M. S., Barton, M. A., \& Lord, F. M. (1983). LOGIST user's guide. Princeton NJ: Educational Testing Service.

\section{A}

Send requests for reprints or further information to Michelle Liou, Institute of Statistical Science, Academia Sinica, Taipei 11529, Republic of China. 